\title{
Hepatocellular adenomas: recent updates
}

\author{
Haeryoung Kim ${ }^{1}$, Young Nyun Park ${ }^{2}$ \\ ${ }^{1}$ Department of Pathology, Seoul National University Hospital, Seoul National University College of Medicine, Seoul; \\ ²Department of Pathology, Graduate School of Medical Science, Brain Korea 21 Project, Yonsei University College of Medicine, Seoul, Korea
}

Hepatocellular adenoma ( $\mathrm{HCA})$ is a heterogeneous entity, from both the histomorphological and molecular aspects, and the resultant subclassification has brought a strong translational impact for both pathologists and clinicians. In this review, we provide an overview of the recent updates on HCA from the pathologists' perspective and discuss several practical issues and pitfalls that may be useful for diagnostic practice.

Key Words: Hepatocellular adenoma; Classification; Pathology

Received: February 4, 2021 Revised: February 27, 2021 Accepted: February 28, 2021

Corresponding Author: Young Nyun Park, MD, PhD, Department of Pathology, Yonsei University College of Medicine, 50-1 Yonsei-ro, Seodaemun-gu, Seoul 03722, Korea Tel: +82-2-2228-1678, Fax: +82-2-362-0860, E-mail: young0608@yuhs.ac

Hepatocellular adenoma (HCA) is defined as a benign monoclonal proliferation of hepatocytes [1]. It is more prevalent in Western countries, with an incidence of $3-4$ cases $/ 100,000$ in Europe and North America [1], and its incidence is much lower in Asian countries [2-6]. Since the first characterization of the molecular subtypes of HCAs in 2006 [7], HCA has now become an increasingly heterogeneous entity, and this has brought a strong translational impact for pathologists (Table 1) [8-10]. In this review, we will summarize the clinicopathological and molecular characteristics of the various subtypes of HCA, and discuss the various pitfalls in the pathological diagnosis of HCA.

\section{GENERAL CLINICOPATHOLOGICAL FEATURES OF HEPATOCELLULAR ADENOMA}

The typical patient is female and of reproductive age (15-50 years), while HCAs are relatively rare in men, children and elderly patients ( $>65$ years). The major risk factors for HCA include oral contraceptive use, obesity, metabolic syndrome, alcohol intake and use of anabolic steroids. Other conditions associated with HCA development include glycogen storage diseases (especially types 1 and 3), galactosemia, tyrosinemia, familial polyposis coli, polycystic ovary syndrome and $\beta$-thalassemia. Interestingly, a few series from Asian countries have demonstrated a higher inci- dence of HCA in men, and the incidence of oral contraceptive use was lower in female patients $[2,3,5]$. The main clinical implications of HCA include the risk of bleeding, especially in larger tumors, and the risk of malignant transformation to hepatocellular carcinoma (HCC). The risk of HCC development depends on the subtype, being the highest in $\beta$-catenin-activated HCAs (B-HCAs). The general indications for surgical management of HCAs include male gender, large size $(>5 \mathrm{~cm})$, interval growth during follow up on imaging, and atypical pathological features (e.g., atypical cytoarchitectural features, presence of $\beta$-catenin activation).

In general, HCAs are grossly well demarcated but non-encapsulated, and the color varies from pale yellow-tan to bile-stained depending on the histology. Hemorrhage or peliosis may be present. HCAs are more frequently solitary lesions; however, multiple HCAs may occur, and the term "adenomatosis" is used when there are 10 or more HCAs. In cases of multiple HCAs, most cases demonstrate multiple HCAs of the same molecular subtype, although some tumors from the same patient may belong to different subgroups of HCA [11]. The size is variable, ranging from $1 \mathrm{~cm}$ to as large as $30 \mathrm{~cm}$. Unlike HCCs, the background liver is typically non-cirrhotic, although advanced stage fibrosis may be present in the setting of alcoholic liver disease, metabolic syndrome or vascular disorders. Histologically, the tumor cells resemble he- 
Table 1. Summary of the clinicopathological and molecular characteristics of different HCA subtypes

\begin{tabular}{|c|c|c|c|c|}
\hline \multirow{2}{*}{ Subtype (frequency, \%) - } & \multicolumn{4}{|c|}{ Characteristic features } \\
\hline & Molecular & Clinical & Histopathological & Immunohistochemical \\
\hline $\begin{array}{l}\text { HNF1A-inactivated HCA } \\
(30 \%-40 \%)\end{array}$ & $\begin{array}{l}\text { HNF1A inactivating mutations } \\
\text { (germline 10\%, somatic 90\%) }\end{array}$ & $\begin{array}{l}\text { Female, obesity, MODY3, } \\
\text { adenomatosis }\end{array}$ & Diffuse steatosis & LFABP expression loss \\
\hline $\begin{array}{l}\text { Inflammatory HCA } \\
(40 \%-50 \%)\end{array}$ & $\begin{array}{l}\text { gp130/IL6ST, FRK, STAT3, GNAS, } \\
\text { JAK1 mutations }\end{array}$ & $\begin{array}{l}\text { Obesity, metabolic syndrome, } \\
\text { alcohol, oral contraceptives }\end{array}$ & $\begin{array}{l}\text { Sinusoidal dilatation } \\
\text { Vascular proliferation } \\
\text { Inflammatory cell } \\
\text { infiltration } \\
\text { Ductular reaction } \\
\text { Focal steatosis }\end{array}$ & SAA, CRP expression \\
\hline \multicolumn{5}{|l|}{$\begin{array}{l}\beta \text {-catenin-activated } \\
\text { HCA (10\%) }\end{array}$} \\
\hline $\begin{array}{l}\beta \text {-catenin } \\
\text { (exon 3)-activated } \\
\text { HCA (7\%) }\end{array}$ & $\begin{array}{l}\text { CTNNB1 exon } 3 \text { activating } \\
\text { mutations }\end{array}$ & $\begin{array}{l}\text { Male, young age, anabolic } \\
\text { steroids, glycogen storage } \\
\text { disease, increased risk of } \\
\text { HCC transformation }\end{array}$ & $\begin{array}{l}\text { Cytological and } \\
\text { architectural atypia }\end{array}$ & $\begin{array}{l}\text { Nuclear } \beta \text {-catenin expression } \\
\text { Diffuse strong GS expression }\end{array}$ \\
\hline $\begin{array}{l}\beta \text {-catenin } \\
\quad(\text { exon } 7,8) \text {-activated } \\
\text { HCA }(3 \%)\end{array}$ & $\begin{array}{l}\text { CTNNB1 exon } 7 \text { or } 8 \text { activating } \\
\text { mutations }\end{array}$ & $\begin{array}{l}\text { Low risk of } \mathrm{HCC} \\
\text { transformation }\end{array}$ & - & $\begin{array}{l}\text { Absent/rare nuclear } \beta \text {-catenin } \\
\text { expression } \\
\text { GS expression: absent/weak/ } \\
\text { patchy }\end{array}$ \\
\hline $\begin{array}{l}\beta \text {-catenin-activated } \\
\text { inflammatory HCA } \\
(5 \%-10 \%)\end{array}$ & $\begin{array}{l}\text { gp130/IL6ST, STAT3, FRK, GNAS, } \\
\text { JAK1 mutations + CTNNB1 exon } \\
3 \text { or } 7 / 8 \text { mutations }\end{array}$ & $\begin{array}{l}\text { Similar to inflammatory HCA } \\
\text { Increased risk of HCC } \\
\text { transformation (ex.3) }\end{array}$ & $\begin{array}{l}\text { Similar to inflammatory } \\
\text { HCA } \\
\text { Cytoarchitectural atypia } \\
\text { (ex.3) }\end{array}$ & $\begin{array}{l}\text { SAA, CRP expression } \\
\text { Nuclear } \beta \text {-catenin, diffuse } \\
\text { strong GS expression (ex.3) }\end{array}$ \\
\hline $\begin{array}{l}\text { Sonic hedgehog- } \\
\text { activated HCA (4\%) }\end{array}$ & $\begin{array}{l}\text { INHBE-GL/1 fusion, resulting } \\
\text { in sonic hedgehog pathway } \\
\text { activation }\end{array}$ & Obesity, hemorrhage & Hemorrhage & PTGDS, ASS1 \\
\hline Unclassified HCA (<7\%) & Unknown & - & - & - \\
\hline
\end{tabular}

HCA, hepatocellular adenoma; MODY3, maturity-onset diabetes type 3; LFABP, liver fatty acid binding protein; SAA, serum amyloid A; CRP, C-reactive protein; HCC, hepatocellular carcinoma; GS, glutamine synthetase; PTGDS, prostaglandin D2 synthase; ASS1, argininosuccinate synthase 1.

patocytes, demonstrating eosinophilic or clear cytoplasm, and they are arranged in thin trabeculae. Portal tracts are absent, and thinwalled vascular channels and small arteriolar structures are seen.

\section{RECENT UPDATES ON THE CLASSIFICATION OF HEPATOCELLULAR ADENOMA}

\section{Inflammatory HCA}

Inflammatory HCA (IHCA) is the most common subtype (40\%-50\%) of HCAs, and demonstrates constitutive activation of the interleukin-6/JAK/STAT pathway. Molecular alterations include mutations in gp130/IL6ST (50\%), FRK (10\%), STAT3 (5\%), GNAS (5\%), ROS1 (3\%), and JAK1 (1\%). The main risk factors for IHCA include obesity, metabolic syndrome and alcohol intake.

The main histological features include foci of inflammation, thick arteries, and sinusoidal dilatation (Fig. 1). Congestion, hemorrhage and peliosis may be present. Portal tracts are absent; however, ductular reaction and pseudoportal tracts are frequently seen. Steatosis may be observed and is usually focal. The background liver frequently demonstrates steatosis, which may be attributed to the patient's underlying risk factors (obesity, met- abolic syndrome, alcoholic liver disease etc.).

Immunohistochemistry for serum amyloid A (SAA) and Creactive protein $(\mathrm{CRP})$ may provide important diagnostic clues, as IHCA is characterized by the overexpression of these acute phase reactants via STAT3 activation. SAA and/or CRP expression in IHCA is usually diffuse and strong, and sharply demarcated from the surrounding liver parenchyme. However, it should be noted that the adjacent liver may be focally or even diffusely positive for SAA and/or CRP in some cases, especially when there is marked inflammation or hemorrhage in the background liver, and in the setting of previous embolization [12]. Therefore, the histological context should be taken into account when interpreting SAA/CRP stains and it is important that the staining results are compared with the background liver, preferably also with positive control tissues.

Importantly, about $10 \%$ of IHCA also demonstrate mutations in CTNNB1 (B-IHCA, "mixed" HCA). Although the risk for HCC transformation is generally low in IHCA, the concurrence of strong $\beta$-catenin activation in IHCAs increases the risk of HCC development. Therefore, the addition of $\beta$-catenin and glutamine synthetase (GS) immunohistochemistry is also necessary, in order to identify mixed HCAs (B-IHCA). B-HCAs 

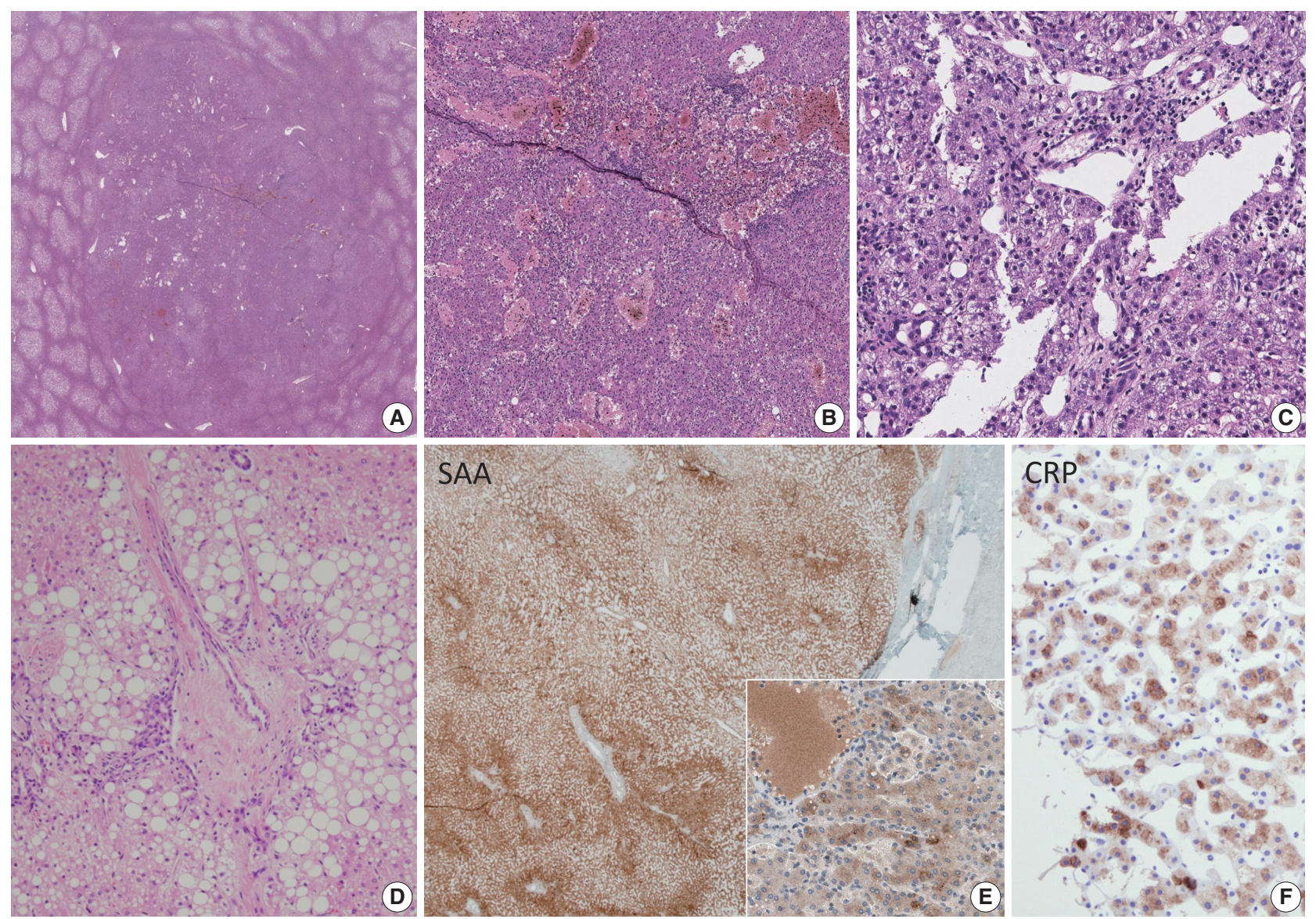

Fig. 1. Inflammatory hepatocellular adenoma. Sinusoidal dilatation, congestion and peliosis is seen in the tumor (A-C). Inflammatory cell infiltration (C), thick arteriolar structures with ductular reaction, resembling portal tracts ("pseudo-portal tracts") and steatosis (D) may be seen in these tumors. Diffuse steatosis may be seen in the background liver (A). The tumor cells express serum amyloid A (E; inset: granular cytoplasmic staining in tumor cells) and C-reactive peptide (F).

are described in more detail in the following section.

\section{$\beta$-catenin-activated HCA}

Approximately $10 \%$ of HCAs demonstrate CTNNB1 mutations/deletions leading to different levels of $\beta$-catenin pathway activation. These tumors are designated as B-HCA. CTNNB1 alterations most often occur in exon $3\left(7 \%, \mathrm{~B}^{\mathrm{ex}}-\mathrm{HCA}\right)$ or in exons 7 and $8\left(3 \%, \mathrm{~B}^{\mathrm{ex}, 8}-\mathrm{HCA}\right)$. Mutations or large deletions in exon 3 most frequently involve the $\beta$-Trcp consensus site (D32-S37, also known as the exon 3 hotspot) and these are associated with high levels of $\beta$-catenin activation and high risk of HCC transformation $[13,14]$. Outside of the $\beta$-Trcp consensus site, T41 and $\mathrm{S} 45$ mutations in exon 3 have also been frequently demonstrated, and these are associated with moderate to weak levels of $\beta$-catenin activation. In contrast, mutations in CTNNB1 exon 7 (K335) and exon 8 (W383, R386, and N387) result in weak $\beta$-catenin activation. The clinical and histopathological features of $\mathrm{B}^{\mathrm{ex}, 8}$-HCA are still unclear, although the risk of HCC development appear to be low in these tumors, unlike the $B^{\text {ex3 }}$-HCAs $[10,13,14]$. CTNNB1 alterations may also occur in a subset of IHCAs (B-IHCA or "mixed" HCA, 5\%-10\%).

The characteristic clinical features associated with $\mathrm{B}^{\mathrm{e} \times 3}-\mathrm{HCAs}$ include male gender, a history of androgen administration and underlying glycogen storage disease. These tumors are usually solitary and rarely multiple. Mild cytological atypia may be observed in $\mathrm{B}^{\mathrm{ex}}$ - $\mathrm{HCAs}$, and architectural atypia, including mild trabecular thickening, small cell change or pseudoglandular structures, is also frequently seen (Fig. 2). Bile or lipofuscin pigments are frequently observed in the tumor cells; the lipofuscin pigmentation may be very prominent in some HCAs [15]. Most importantly, as $B^{\mathrm{ex}}$-HCAs are associated with high risk of malignant transformation to HCC, the practical implication of this subtype of HCA is in excluding the possibility of well-differentiated HCC. This differential diagnosis is discussed later. 

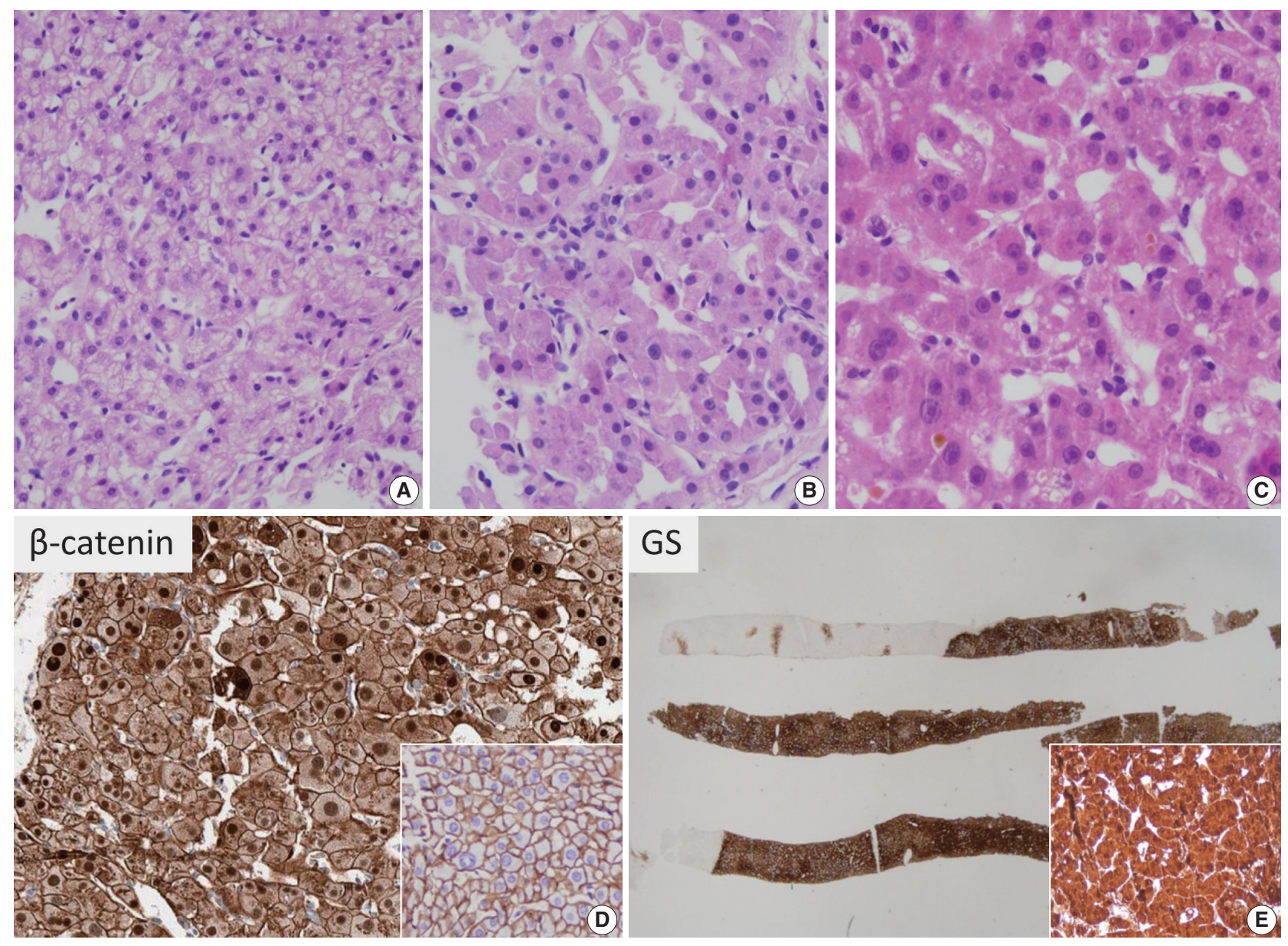

Fig. 2. $\beta$-catenin-activated hepatocellular adenoma (B-HCA). There is mild trabecular thickening $(A)$ and pseudoglandular structures $(B, C)$. Mild cytological atypia is present (A-C). Cholestasis may be seen (C). On immunohistochemistry, B-HCA with strong $\beta$-catenin activation (Bex3-HCA) demonstrates nuclear $\beta$-catenin expression (D), instead of the normal membranous pattern (inset), and diffuse homogeneous glutamine synthetase expression is seen in such tumors ( $E$, inset: higher-power view).

Although direct sequencing of the CTNNB1 gene would be the most definitive means of characterizing the molecular subtype of a presumed B-HCA, this is not feasible in most clinical practices. Fortunately, immunohistochemical stains for $\beta$-catenin and GS have been demonstrated to be good surrogate markers reflecting CTNNB1 status. Nuclear $\beta$-catenin expression and diffuse strong homogeneous GS expression are the typical immunohistochemical features of B-HCAs with strong $\beta$-catenin activation (non-S45 $B^{\text {ex3 }}$-HCA). Interestingly, GS expression patterns have been recently demonstrated to reflect the mutational status of CTNNB1 (Fig. 3). Diffuse homogeneous expression (strong GS expression in $>90 \%$ of tumor cells) has been demonstrated in $\mathrm{B}^{\text {ex }}$-HCAs with mutations or large deletions in the D32-S37 hotspot of CTNNB1 exon 3 ( $\beta$-Trcp consensus site). In contrast, $B^{\text {ex3 }}$-HCAs with CTNNB1 exon 3 T41 or $\$ 45$ mutations, which have been associated with moderate $\beta$-catenin activation, dem- onstrate diffuse heterogeneous GS expression (50\%-90\% of tumor cells expressing GS in a starry-sky pattern). $B^{\text {ex } 7,8}$-HCAs lack the immunohistochemical features of strong $\beta$-catenin activation (i.e., diffuse strong GS expression, nuclear $\beta$-catenin expression). Weak patchy GS staining in addition to the "normal" perivenular pattern has been frequently seen in association with weak $\beta$-catenin activation (absent or rare nuclear $\beta$-catenin), which is common in $\mathrm{B}^{\mathrm{ex} 7,8}$-HCAs, but also rarely seen in $\mathrm{B}^{\mathrm{ex} 3}$-HCAs with $\$ 45$ mutations. Interestingly, GS accentuation and discontinuous band-like GS staining at the tumor border has been described in $\mathrm{B}^{\mathrm{ex} 3}$-HCAs with $S 45$ mutations and $B^{\text {ex } 7,8}$-HCAs, respectively $[16,17]$. These expression patterns are different from the "map-like" GS pattern of focal nodular hyperplasia (FNH), which is described later. In contrast, GS expression in the normal liver has a perivenular distribution ("normal" pattern), where GS expression is limited to 1 to 3 layers of hepatocytes around the central vein. 


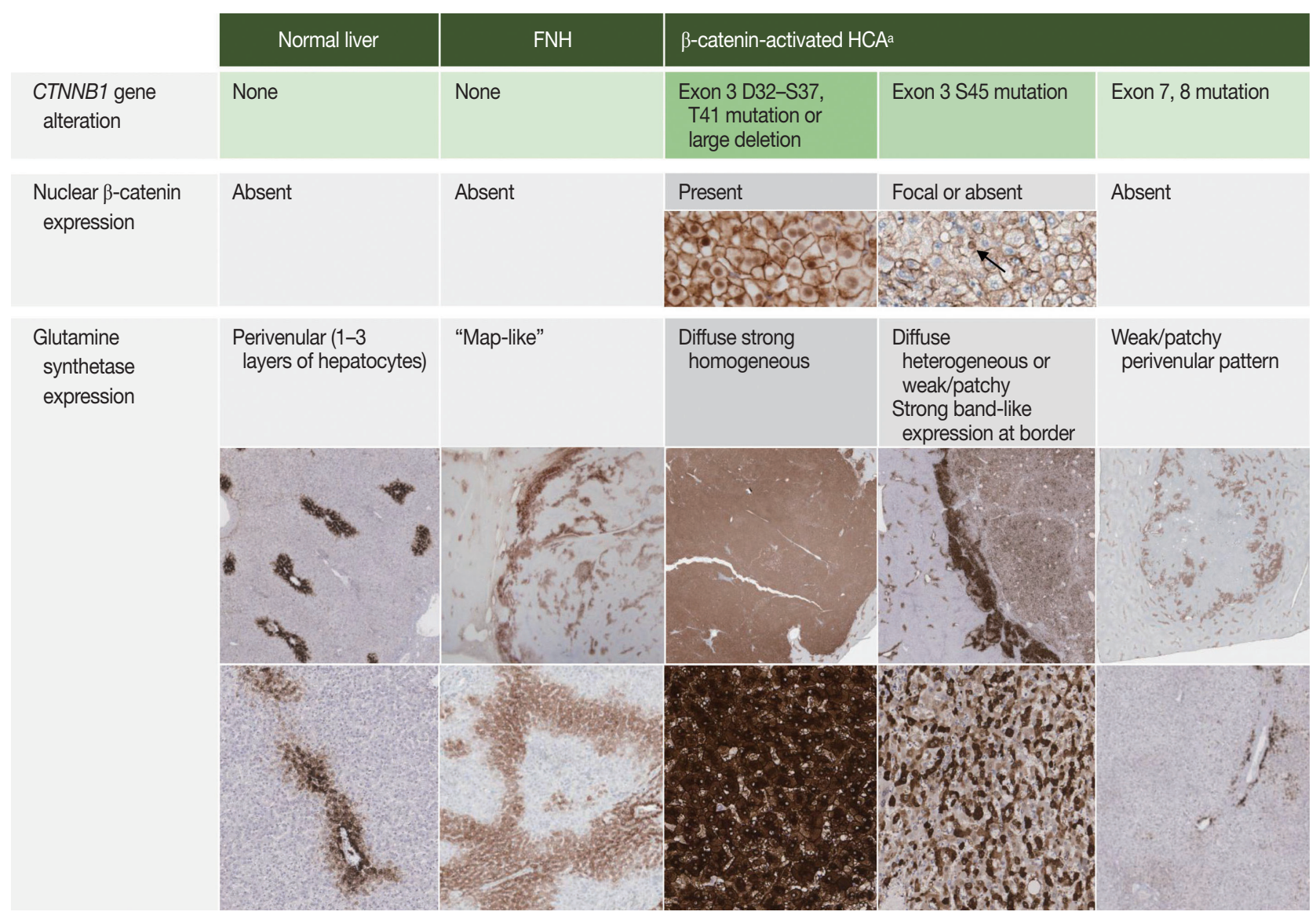

Fig. 3. Immunohistochemical correlates of CTNNB1 alteration status in $\beta$-catenin-activated HCA, FNH and normal liver. FNH, focal nodular

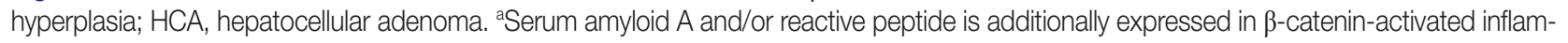
matory HCA.

\section{HNF1A-inactivated HCA}

HNF1A-inactivated HCAs (H-HCA) constitute 30\%-35\% of HCAs and are characterized by inactivating mutations in HNF1A, which encodes hepatocyte nuclear factor $1 \alpha$. In the majority (90\%) of H-HCAs, the HNF1A mutation is somatic and biallelic, while the remaining $10 \%$ comprise $\mathrm{H}-\mathrm{HCAs}$ with germline HNF1A mutations. The latter is frequently associated with maturity-onset diabetes type 3 (MODY3) and the presence of adenomatosis. H-HCAs have also been reported in the background of vascular abnormalities and congenital hepatic fibrosis [18-20].

Histologically, H-HCAs typically demonstrate diffuse steatosis in the tumor cells (Fig. 4), although some cases may show minimal or no steatosis, especially those arising in hepatic vascular disorders $[20,21]$. The tumor cells demonstrate no significant nuclear atypia and they are usually arranged in thin trabeculae, although occasional pseudoglandular structures may be observed. Inflammatory cell infiltration is not a characteristic of H-HCA.
Immunohistochemically, the tumor cells demonstrate loss of liver fatty acid binding protein (LFABP) expression. H-HCAs demonstrate a low risk of HCC transformation compared to other types.

\section{Unclassified HCA and sonic hedgehog-activated HCA}

By definition, unclassified HCA (U-HCA) lacks the characteristics of other subtypes. The frequency of U-HCA (5\%-10\%) is decreasing, as HCAs belonging to this group are being increasingly characterized as specific subtypes. Sonic hedgehog-activated HCA (sh-HCA) is an emerging subtype of HCA with distinct clinicopathological features, including increased bleeding risk. sh-HCA has been demonstrated to account for $\sim 4 \%$ of HCAs that were previously classified as U-HCA [10]. Molecular features of sh-HCA include INBHE-GLI1 fusion and overexpression of GLI1, which is the key transcription factor of the sonic hedgehog pathway. Recently, potential surrogate immunohistochemical marker candidates have received attention, including 

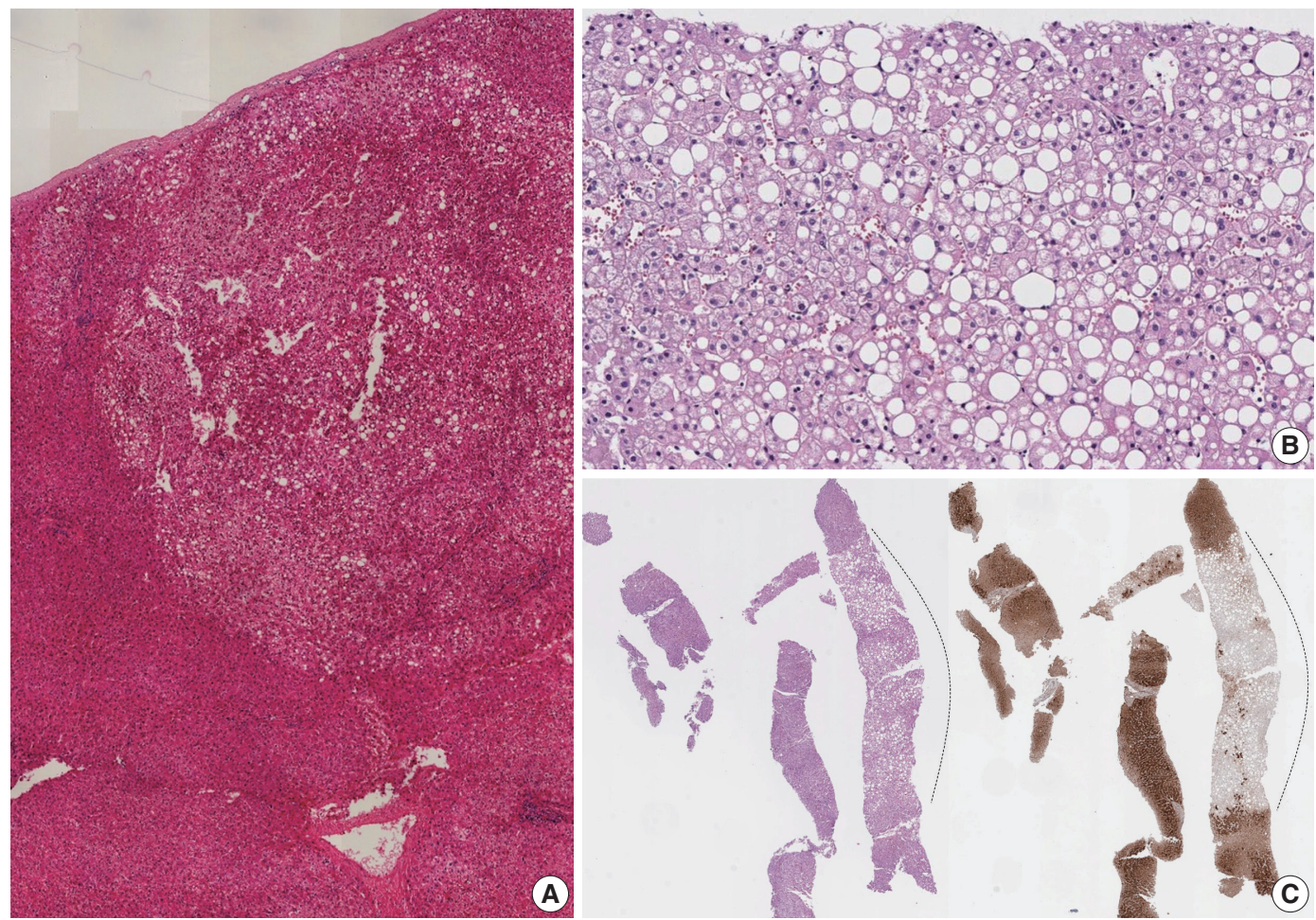

Fig. 4. HNF1A-inactivated hepatocellular adenoma. Diffuse steatosis is seen in the tumor, compared to the surrounding hepatic parenchyme $(\mathrm{A}-\mathrm{C})$. There is no significant cytological or architectural atypia on higher-power magnification (B). Immunohistochemically, the tumor displays loss of liver fatty acid-binding protein expression (C) (dotted line: tumor).

lipocalin-type prostaglandin D2 synthase and argininosuccinate synthase 1 [22-25].

\section{COMMON PRACTICAL ISSUES FOR PATHOLOGISTS}

\section{Atypia in HCAs: HCCs or atypical hepatocellular neoplasms?}

In practice, pathologists are often faced with having to distinguish between HCA and well-differentiated HCC, which is a difficult challenge especially on needle biopsies. Features that favor the possibility of HCC include cytological atypia which is more than minimal and patchy, thickened hepatocyte trabeculae, frequent pseudoglandular structures, cholestasis, small cell change and loss of reticulin staining (Fig. 5). Caution should be exercised when interpreting reticulin loss, as focal reticulin loss in an HCA is acceptable when there is marked steatosis [26]. Conversely, well differentiated HCCs show only a partial loss of reticulin [27]. Identification of stromal invasion or a nodule-in-nodule growth pattern adds more confidence to the diagnosis of HCC. In the latter case, the outer and inner nodules are usually composed of HCA and HCC, respectively. The 3-marker panelglypican-3, heat shock protein 70 (HSP70) and GS-which is commonly used in the differential diagnosis between dysplastic nodules and HCC may be also be helpful for the discrimination between HCA and HCC [27-29]. However, as the challenging question of "HCA versus HCC" usually arises in the context of B-HCAs with strong/moderate $\beta$-catenin activation that express diffuse GS, the additional expression of glypican-3 and/or HSP70 would be more helpful in practice [29]. Although HCC often demonstrates increased sinusoidal capillarization compared to HCA (highlighted by CD34), this is a relative increase without definite cut-off values, and therefore CD34 immunohistochemistry on its own has limited use in the differential diagnosis.

The terms "atypical hepatocellular neoplasm (AHN)" and "hepatocellular neoplasm of uncertain malignant potential (HUMP)" have been proposed for hepatocellular neoplasms that demonstrate features atypical for HCA but insufficient for a confident diagnosis of HCC [30-32]. These terms have been coined to emphasize the borderline characteristics of these tumors, including increased risk for HCC development, and to convey to the clinicians the necessity for surgical intervention or at least close follow-up. There are currently no widely accepted guidelines on when to use these terms, although the following features have been consistently described in AHN/HUMPs: (1) morpho- 

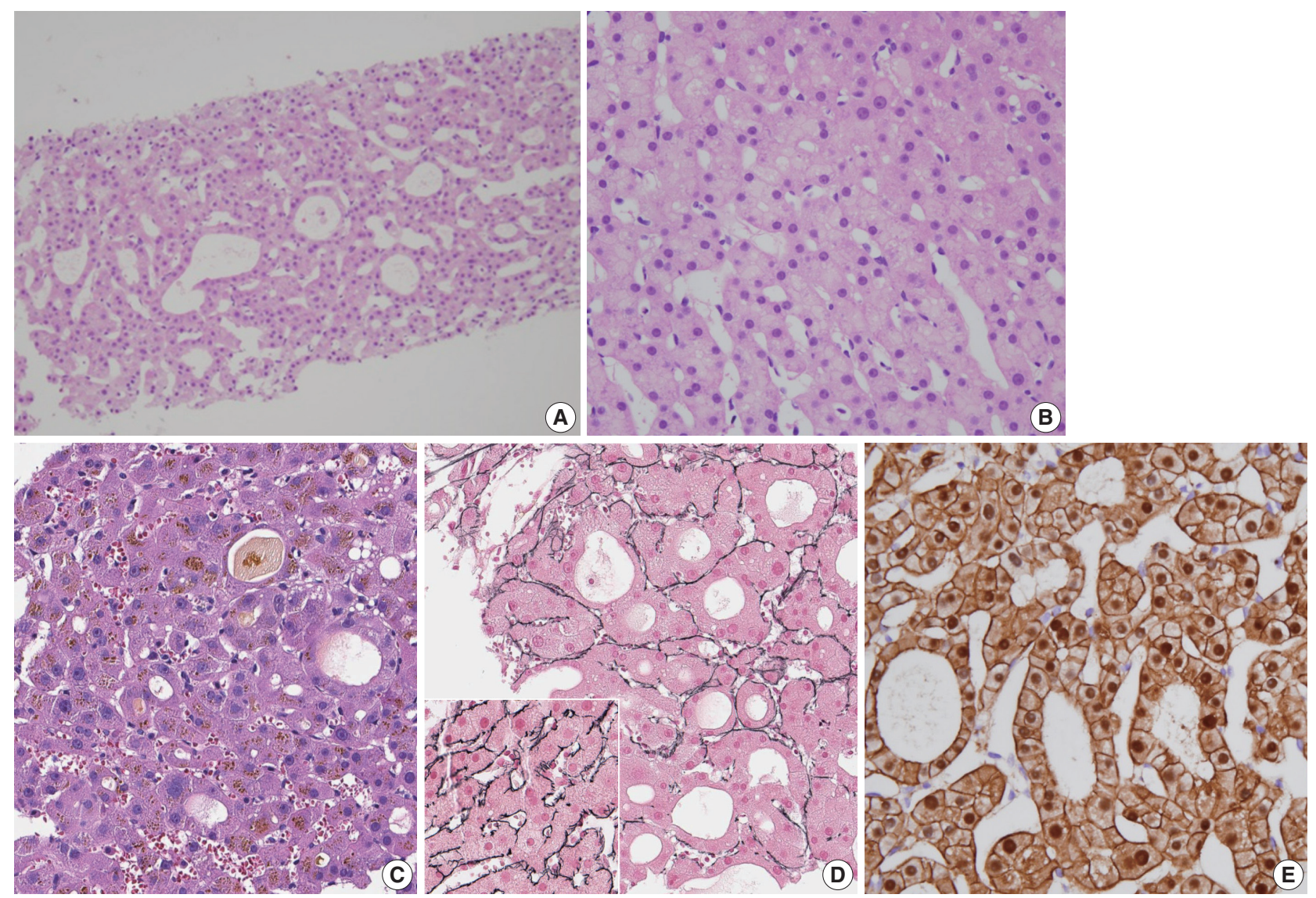

Fig. 5. Atypia in hepatocellular neoplasms. When features atypical for hepatocellular adenomas, such as frequent pseudoglandular structures (A, C-E), nuclear atypia (B, C) and focal reticulin loss (D; inset: preserved reticulin framework for comparison), are identified, the differential diagnosis should be between atypical hepatocellular neoplasm and well-differentiated hepatocellular carcinoma. When these features are only focally present $(<5 \%)$, the terminology of atypical hepatocellular neoplasm may be used. Nuclear $\beta$-catenin expression is often identified in such cases (E).

logical atypia (nuclear atypia, small cell change, pseudoglandular structures, loss of reticulin fibers) that is only focal $(<5 \%$ of the tumor), (2) presence of $\beta$-catenin activation or CTNNB1 mutation, and (3) atypical demographic features (e.g., male, age > 50 years) [30-33]. Of note, Evason et al. [31] demonstrated similar cytogenetic alterations to HCC in their series of AHNs, and also documented recurrence and metastasis in AHNs with $\beta$-catenin activation, suggesting that these tumors may actually represent an extremely well-differentiated group of HCCs [31]. The presence of nuclear $\beta$-catenin expression, diffuse GS expression, or indeterminate diffuse GS expression in hepatocellular neoplasms (thus, suggestive of $\mathrm{B}^{\mathrm{ex}}$ - $\mathrm{HCA}$ ) have been suggested as criteria for AHN, especially on biopsied specimens [30]. This would guide the clinician to surgically resect the nodule in question, rather than subjecting the patient to follow up, which is important as (1) an overt HCC may be present elsewhere in the nodule, and (2) $\mathrm{B}^{\mathrm{e} \times 3}-\mathrm{HCA}$ have been associated with increased risk of HCC development. On resected specimens, the diagnosis of B-HCA may be made when the degree of cytoarchitectural atypia is insufficient for HCC, although some still prefer to use AHN in this setting [30].

\section{IHCA vs. FNH vs. mass effect}

The presence of ductular reaction, pseudoportal tracts and abnormal thick-walled vessels may result in the resemblance of IHCA to FNH. There is indeed a significant histological overlap between FNH and IHCA, and in fact, lesions previously described as telangiectatic FNH have now been reclassified as IHCA on the basis of molecular studies [34-36]. Discriminating between IHCA and FNH on a needle biopsy is a common difficult challenge for pathologists. $\mathrm{FNH}$ is a reactive polyclonal proliferation of hepatocytes in response to vascular abnormalities. Grossly, it is a well-demarcated multinodular mass with a central depressed stellate scar and radiating fibrous septa. Microscopically, central 
fibrous scars and radiating fibrous septa with abnormal vasculature, including eccentrically thickened vascular walls, and marked ductular reaction are the typical findings. The hepatocytes demonstrate no significant atypia, and the hepatocyte plates are 1-3 cells thick. The nodularity, fibrous septa and ductular reaction may also be seen in IHCAs; in these situations, immunohistochemistry for GS, SAA, and CRP may be helpful. FNH demonstrates a remarkable "map-like" GS expression pattern, characterized by broad bands of hepatocytes that strongly express GS, and these bands appear to anastomose together to produce a complex lace-like or map-like architecture (Figs. 3, 6) [37,38].
On biopsies, the GS expression appears as patchy broad bands of strong GS expression, which contrast with the punctate or linear perivenular GS pattern in the adjacent normal parenchyme (Fig. 6). Nuclear $\beta$-catenin staining is absent in FNH, and SAA and CRP are usually negative in FNH. Thus, SAA and/or CRP expression and absence of "map-like" GS expression pattern strongly supports a diagnosis of IHCA over FNH.

Mass effect may appear as sinusoidal dilatation and ductular reaction in the non-lesional hepatic parenchyme, which may lead to an erroneous diagnosis of IHCA [39]. The presence of portal tract edema and fibrosis with neutrophilic infiltration should alert
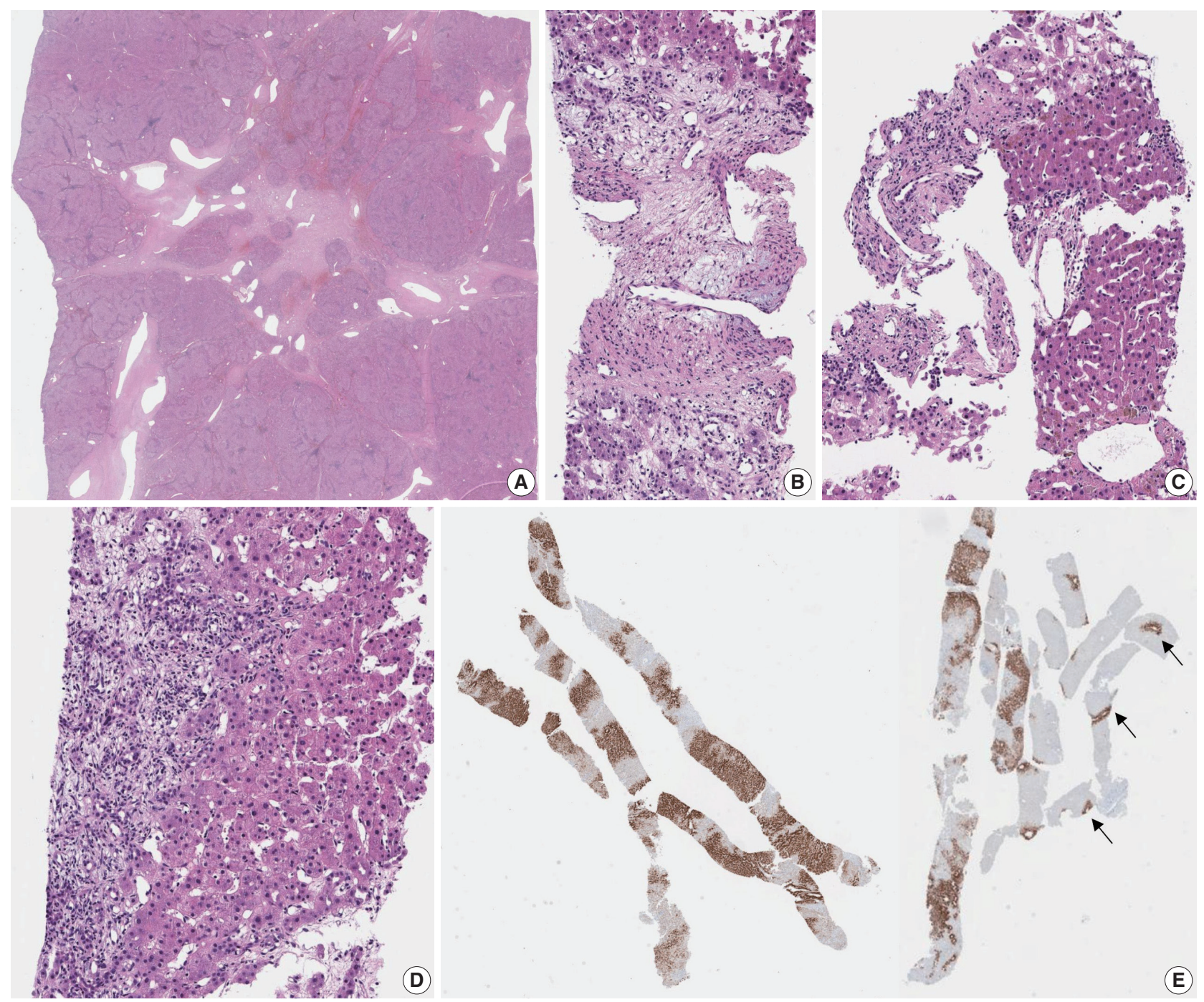

Fig. 6. Focal nodular hyperplasia. A typical low power view of a resected case (A). A central stellate scar with radiating fibrous septa is seen, containing multiple irregular-shaped vascular structures. When needle biopsy specimens are obtained from the central scar or fibrous septa $(B, C)$, it is easy to identify the abnormal vascular channels with varying calibers, shape and irregular walk thickness. Sometimes biopsies include the peripheral portions without the obvious vascular anomalies; instead there is at least mild hepatocellular nodularity with ductular reaction (D). Glutamine synthetase immunohistochemistry demonstrates broad bands of GS-expressing hepatocytes (E), which corresponds to the map-like pattern in Fig. 3, in contrast to the normal perivenular pattern (arrows). 
the pathologist to the possibility that the biopsy core was obtained from the perilesional parenchyme and clinicoradiological correlation is mandatory. Recognizing the presence of evenly spaced portal tracts and central veins, perhaps with the help of trichome and GS stains, may also be helpful in suggesting the non-neoplastic nature of the biopsied tissue.

\section{Diagnostic pitfalls in the interpretation of immunohistochemical stain results}

GS, $\beta$-catenin, SAA, CRP, and LFABP are indeed helpful immunohistochemical tools for subtyping HCAs and/or discriminating between HCA and FNH. However, they should also be used with care and in the correct context. SAA and CRP expression have been reported in $17 \%$ and $~ 50 \%$ of HCCs, respectively, and loss of LFABP expression has been demonstrated in 16\%-25\% of HCCs [40-42]. In addition, as previously mentioned, the background liver may show focal expression of SAA or CRP, especially in the setting of inflammation or hemorrhage; therefore, positivity for these markers should be interpreted carefully, taking the histology into account. Moreover, some cirrhotic nodules may express SAA and/or CRP, such as in the SAA-positive nodules in alcoholic cirrhosis [43]. Therefore, these markers are not specific for HCA.

GS expression is a well-known component of the 3-marker panel (glypican-3, HSP70, and GS) for diagnosing HCCs $[28,44]$. Therefore, immunostains used for subtyping HCAs should only be used after an initial impression of HCA has been made, and not for distinguishing between HCA and HCC.

\section{CONCLUSION}

Although simply defined as a benign neoplasm of hepatocellular origin, HCA is a heterogeneous entity from both molecular and histomorphological aspects. The molecular classification of HCA has helped to increase our understanding of the biology of these tumors. More importantly for pathologists, the molecular classification has also helped to characterize the morphological and immunohistochemical features in HCAs in detail. The association between CTNNB1 mutation status and the clinical behavior of HCA brings enormous translational impact, as the presence of strong $\beta$-catenin activation may influence management decisions in favor of surgical treatment. As for H-HCAs, the identification of individuals with liver adenomatosis composed of H-HCA should prompt molecular testing for germline $H N$ F1A mutation status, in addition to familial screening for liver adenomatosis. It is also possible that additional subtypes of HCA will be identified in the future.

\section{Ethics Statement}

Not applicable.

\section{Availability of Data and Material}

Data sharing not applicable to this article as no datasets were generated or analyzed during the study.

\section{Code Availability}

Not applicable.

\section{ORCID}

Haeryoung Kim https://orcid.org/0000-0002-4205-9081 Young Nyun Park https://orcid.org/0000-0003-0357-7967

\section{Author Contributions}

Conceptualization: HK, YNP. Funding acquisition: HK, YNP. Study supervision: HK, YNP. Writing—original draft: HK. Writing—review \& editing: HK, YNP. Approval of final manuscript: HK, YNP.

\section{Conflicts of Interest}

H.K., a contributing editor of the Journal of Pathology and Translational Medicine, was not involved in the editorial evaluation or decision to publish this article. Remaining author has declared no conflicts of interest.

\section{Funding Statement}

This work was supported by the National Research Foundation of Korea (NRF) grant funded by the Korea government (NRF-2019R1A2C2010056, NRF-2016R1D1A1A09919042 to HK; NRF-2020R1A2B5B01001646 to YNP) and by the Bio and Medical Technology Development Program of the NRF funded by the Ministry of Science and ICT (MSIT) (NRF-2016M3 A9D5A01952416) (YNP).

\section{References}

1. Bioulac-Sage P, Kakar S, Nault JC. Hepatocellular adenoma. In: WHO classification of tumours: digestive system tumours. 5th ed. Lyon: International Agency for Research on Cancer, 2019; 224-8.

2. Lin H, van den Esschert J, Liu C, van Gulik TM. Systematic review of hepatocellular adenoma in China and other regions. J Gastroenterol Hepatol 2011; 26: 28-35.

3. Sasaki M, Yoneda N, Kitamura S, Sato Y, Nakanuma Y. Characterization of hepatocellular adenoma based on the phenotypic classification: the Kanazawa experience. Hepatol Res 2011; 41: 982-8.

4. Sasaki M, Nakanuma Y. Overview of hepatocellular adenoma in Japan. Int J Hepatol 2012; 2012: 648131.

5. Liu HP, Zhao Q, Jin GZ, et al. Unique genetic alterations and clinicopathological features of hepatocellular adenoma in Chinese population. Pathol Res Pract 2015; 211: 918-24.

6. Huang WC, Liau JY, Jeng YM, et al. Hepatocellular adenoma in Taiwan: distinct ensemble of male predominance, overweight/obesity, and inflammatory subtype. J Gastroenterol Hepatol 2020; 35: 680-8.

7. Zucman-Rossi J, Jeannot E, Nhieu JT, et al. Genotype-phenotype correlation in hepatocellular adenoma: new classification and relationship with HCC. Hepatology 2006; 43: 515-24.

8. Nault JC, Bioulac-Sage P, Zucman-Rossi J. Hepatocellular benign tumors-from molecular classification to personalized clinical care. Gastroenterology 2013; 144: 888-902.

9. Scoazec JY. Hepatocellular adenomas: one step beyond. Gut 2019; 
68: 1140-2.

10. Nault JC, Couchy G, Balabaud C, et al. Molecular classification of hepatocellular adenoma associates with risk factors, bleeding, and malignant transformation. Gastroenterology 2017; 152: 880-94.

11. Nault JC, Zucman Rossi J. Molecular classification of hepatocellular adenomas. Int J Hepatol 2013; 2013: 315947.

12. Shin JH, Yu E, Kim EN, Kim CJ. C-reactive protein overexpression in the background liver of hepatitis B virus-associated hepatocellular carcinoma is a prognostic biomarker. J Pathol Transl Med 2018; 52: 267-74.

13. Rebouissou S, Franconi A, Calderaro J, et al. Genotype-phenotype correlation of CTNNB1 mutations reveals different $\beta$-catenin activity associated with liver tumor progression. Hepatology 2016; 64: 2047-61.

14. Pilati C, Letouze E, Nault JC, et al. Genomic profiling of hepatocellular adenomas reveals recurrent FRK-activating mutations and the mechanisms of malignant transformation. Cancer Cell 2014; 25: 428-41.

15. Mounajjed T, Yasir S, Aleff PA, Torbenson MS. Pigmented hepatocellular adenomas have a high risk of atypia and malignancy. Mod Pathol 2015; 28: 1265-74.

16. Bioulac-Sage P, Sempoux C, Balabaud C. Hepatocellular adenoma: classification, variants and clinical relevance. Semin Diagn Pathol 2017; 34: 112-25.

17. Cappellen D, Balabaud C, Bioulac-Sage P. A difficult case of betacatenin-mutated hepatocellular adenoma: a lesson for diagnosis. Histopathology 2019; 74: 355-7.

18. Ibarrola C, Castellano VM, Colina F. Focal hyperplastic hepatocellular nodules in hepatic venous outflow obstruction: a clinicopathological study of four patients and 24 nodules. Histopathology 2004; 44: 172-9.

19. Paradis V, Bioulac-Sage P, Balabaud C. Congenital hepatic fibrosis with multiple HNF1alpha hepatocellular adenomas. Clin Res Hepatol Gastroenterol 2014; 38: e115-6.

20. Lee Y, Park H, Lee K, Lee Y, Lee K, Kim H. Multiple hepatocyte nuclear factor 1A (HNF1A)-inactivated hepatocellular adenomas arising in a background of congenital hepatic fibrosis. J Pathol Transl Med 2021; 55: 154-8.

21. Sempoux C, Balabaud C, Paradis V, Bioulac-Sage P. Hepatocellular nodules in vascular liver diseases. Virchows Arch 2018; 473: 33-44.

22. Frulio N, Balabaud C, Laurent C, Trillaud H, Bioulac-Sage P. Unclassified hepatocellular adenoma expressing ASS1 associated with inflammatory hepatocellular adenomas. Clin Res Hepatol Gastroenterol 2019; 43: e63-7.

23. Henriet E, Abou Hammoud A, Dupuy JW, et al. Argininosuccinate synthase 1 (ASS1): a marker of unclassified hepatocellular adenoma and high bleeding risk. Hepatology 2017; 66: 2016-28.

24. Nault JC, Couchy G, Caruso S, et al. Argininosuccinate synthase 1 and periportal gene expression in sonic hedgehog hepatocellular adenomas. Hepatology 2018; 68: 964-76.

25. Sala M, Gonzales D, Leste-Lasserre T, et al. ASS1 overexpression: a hallmark of sonic Hedgehog hepatocellular adenomas: recommendations for clinical practice. Hepatol Commun 2020; 4: 809-24.

26. Singhi AD, Jain D, Kakar S, Wu TT, Yeh MM, Torbenson M. Reticulin loss in benign fatty liver: an important diagnostic pitfall when considering a diagnosis of hepatocellular carcinoma. Am J Surg Pathol 2012; 36: 710-5.

27. Kim H, Park YN. Role of biopsy sampling for diagnosis of early and progressed hepatocellular carcinoma. Best Pract Res Clin Gas- troenterol 2014; 28: 813-29.

28. Di Tommaso L, Destro A, Seok JY, et al. The application of markers (HSP70 GPC3 and GS) in liver biopsies is useful for detection of hepatocellular carcinoma. J Hepatol 2009; 50: 746-54.

29. Lagana SM, Salomao M, Bao F, Moreira RK, Lefkowitch JH, Remotti HE. Utility of an immunohistochemical panel consisting of glypican-3, heat-shock protein-70, and glutamine synthetase in the distinction of low-grade hepatocellular carcinoma from hepatocellular adenoma. Appl Immunohistochem Mol Morphol 2013; 21: 170-6.

30. Choi WT, Kakar S. Atypical hepatocellular neoplasms: review of clinical, morphologic, immunohistochemical, molecular, and cytogenetic features. Adv Anat Pathol 2018; 25: 254-62.

31. Evason KJ, Grenert JP, Ferrell LD, Kakar S. Atypical hepatocellular adenoma-like neoplasms with beta-catenin activation show cytogenetic alterations similar to well-differentiated hepatocellular carcinomas. Hum Pathol 2013; 44: 750-8.

32. Kakar S, Evason KJ, Ferrell LD. Well-differentiated hepatocellular neoplasm of uncertain malignant potential: proposal for a new diagnostic category: reply. Hum Pathol 2014; 45: 660-1.

33. Roncalli M, Sciarra A, Tommaso LD. Benign hepatocellular nodules of healthy liver: focal nodular hyperplasia and hepatocellular adenoma. Clin Mol Hepatol 2016; 22: 199-211.

34. Laumonier H, Frulio N, Laurent C, Balabaud C, Zucman-Rossi J, Bioulac-Sage P. Focal nodular hyperplasia with major sinusoidal dilatation: a misleading entity. BMJ Case Rep 2010; 2010: bcr0920103311.

35. Paradis V, Benzekri A, Dargere D, et al. Telangiectatic focal nodular hyperplasia: a variant of hepatocellular adenoma. Gastroenterology 2004; 126: 1323-9.

36. Bioulac-Sage P, Rebouissou S, Sa Cunha A, et al. Clinical, morphologic, and molecular features defining so-called telangiectatic focal nodular hyperplasias of the liver. Gastroenterology 2005; 128: 1211-8.

37. Bioulac-Sage P, Cubel G, Taouji S, et al. Immunohistochemical markers on needle biopsies are helpful for the diagnosis of focal nodular hyperplasia and hepatocellular adenoma subtypes. Am J Surg Pathol 2012; 36: 1691-9.

38. Joseph NM, Ferrell LD, Jain D, et al. Diagnostic utility and limitations of glutamine synthetase and serum amyloid-associated protein immunohistochemistry in the distinction of focal nodular hyperplasia and inflammatory hepatocellular adenoma. Mod Pathol 2014; 27: 62-72.

39. Agostini-Vulaj D, Sharma AK, Findeis-Hosey JJ, McMahon LA, Gonzalez RS. Distinction between inflammatory hepatocellular adenoma and mass effect on liver sampling. Hum Pathol 2017; 61: 105-10.

40. Kim H, Lee H, Park YN. Loss of liver fatty acid binding protein expression in hepatocellular carcinomas is associated with a decreased recurrence-free survival. J Liver Cancer 2015; 15: 30-5.

41. Liu L, Shah SS, Naini BV, et al. Immunostains used to subtype hepatic adenomas do not distinguish hepatic adenomas from hepatocellular carcinomas. Am J Surg Pathol 2016; 40: 1062-9.

42. Torbenson M. Hepatic adenomas: classification, controversies, and consensus. Surg Pathol Clin 2018; 11: 351-66.

43. Sasaki M, Yoneda N, Kitamura S, Sato Y, Nakanuma Y. A serum amyloid A-positive hepatocellular neoplasm arising in alcoholic cirrhosis: a previously unrecognized type of inflammatory hepatocellular tumor. Mod Pathol 2012; 25: 1584-93.

44. Di Tommaso L, Franchi G, Park YN, et al. Diagnostic value of HSP70, glypican 3, and glutamine synthetase in hepatocellular nodules in cirrhosis. Hepatology 2007; 45: 725-34. 